\title{
BLACK BOX CLOSED LOOP ROBOT MANIPULATOR SYSTEM IDENTIFICATION
}

\author{
Aziz* I. Said and Ashraf** S. Awad,
}

\begin{abstract}
The paper discusses experimental identification of one joint of a hand made, two degrees of freedom robot manipulator, including flexibilities, under feedback. A black box system model is identified from the input-output data. Both linear, OE (Output Error) and non-linear structure (multilayer perceptrons neural network) models are treated and applied. A Levenberg-Marquardt algorithm is implemented to generate our NNARX model. As regressors two past inputs and two past outputs are chosen. Furthermore network architecture is chosen with 5 hidden tanh units and one linear output unit. Fit criteria shows that the linear model has severe problems. Validation of the trained non-linear network looks quite satisfactory, and it is definitely better than the linear model. Experience has shown that regularization is helpful when pruning neural networks. A remarkable improvement in performance, when using long instead of short format for choosing neural network weights and Bias, is appreciated.
\end{abstract}

\section{KEY WORDS}

Arms control, ART neural networks, Autoregressive moving average processes, Closed loop systems, Control systems, Correlation, Feedback systems, Identification, Manipulators, Motor drives, Optical position measurement.

\section{NOMENCLATURE}

DC: $\quad$ Direct Current

NNARX: Neural Network Auto Regressive with eXogenous input.

OE: $\quad$ Output Error

\footnotetext{
*Aziz I. Said is a Prof. Dr. with the Department of Electrical Power and Machine, Faculty of Engineering, Ain Shams University. Egypt, Cairo. (e-mail: aziz_said44@yahoo.com).

**Ashraf S. Awad is with Quality Control of Machining production Department, in Egyptian Tank Plant, MF 200, Abo Zabal, Cairo, Egypt. (e-mail: a_awad@hotmail.com ).
} 


\section{INTRODUCTION}

Robot manipulators are basically multi-degree-of-freedom positioning devices. The robot as the "plant to be controlled", is a multi-input / multi-output, highly coupled, non linear mechatronic system. The main challenges in the motion control problem are the complexity of the dynamics, and uncertainties, both parametric and dynamic. Parametric uncertainties arise from imprecise knowledge of the kinematics and dynamics, while dynamic uncertainties arise from joint and link flexibility, actuator dynamics, friction, sensor noise, and unknown environment dynamics. [1]

The most important factors in manufacturing are quality, costs and time. When robots are used to meet these demands it is important to have good models of the robots. To reduce the building costs, the robots are built to be lighter and this results in weaker structures. It is therefore a need for flexible robot models instead of rigid models to keep the same quality and performance of the robot.

We look at an experimental robot system and perform identification of the dynamics. Real data from this robot are used in the identification experiments. The model can be used to improve the control of the robot, especially by utilizing the frequencies around and above the resonance frequency. Another area where it is important to have good dynamic models is fault detection and isolation. We will concentrate the work on the identification part and leave the use of the acquired models to future work.

Identification is not a new topic in the robot community, and a general discussion can for example be found in [2]. At the heart of any estimation problem is to select a suitable model structure. A model structure is a parameterized family of candidate models of some sort, within which the search for a model is conducted. A basic rule in estimation is not to estimate what you already know. In other words, one should utilize prior knowledge and physical insight about the system when selecting the model structure. It is customary to color-code, in shades of grey, the model structure according to what type of prior knowledge has been used:

- White-box models: This is the case when a model is perfectly known; it has been possible to construct it entirely from prior knowledge and physical insight.

- Grey-box models: This is the case when some physical insight is available, but several parameters remain to be determined from observed data.

- Black-box models: No physical insight is available or used, but the chosen model structure belongs to families that are known to have good flexibility and have been "successful in the past".

This paper deals with Black-box models for dynamical systems, for which inputs and outputs can be measured [3]. In this paper the identification of the Robot is performed under strong feedback. The main problem with identification from closed loop data is that the data contain less information about the open loop system. The reason for this is that the purpose of the feedback is to make the closed loop system less sensitive to changes in the open loop system. In Identification for control, the 
objective is to achieve a model that is suited for robust control design. Thus one has to tailor the experiment and preprocessing of data so that the model is reliable in regions where the design process doesn't tolerate significant uncertainties. Other reasons for using closed loop experiments might be that the plant is unstable, or that it contains inherent feedback mechanisms. Another problem that can occur is bias due to inaccurate noise models. The identification is made using the System Identification Toolbox [4].

\section{DESCRIPTION OF THE ROBOT SYSTEM}

This section gives an overview of the physical system used in the Identification experiments. A picture of the manipulator is shown in Fig. 1, It has two axes operating in the vertical plane. Both joints of the robot are driven by permanentmagnet DC motor. The two arms of the robot are $0.17 \mathrm{~m}$ in length. Adaptive control algorithm is implemented in a PC. Both of the joint positions are measured by digital optical incremented encoders [5].

In this paper, we, only, model the motion at first joint axis. That is, one electrical motor, with gears, and the robot arm are modeled. The structure of the robot system is depicted in Fig. 2. The torque generated by the electrical motor is affected only indirectly by the feedback control system.

In this experiment, the reference signal $\theta_{\text {ref, }}$ is generated. The angle of the motor driving the robot arm, " $\theta_{m}$ " is measured, as an output signal. Driving input signal "u" is produced by PC. The sampling interval is $4 \mathrm{~ms}$. The goal is to identify a model of the robot by using $u$ as an input, and $\theta_{m}$ as an output (measured arm angular joint).

\section{DESIRED TRAJECTORY}

The Test trajectory is a circular task space. The corresponding angular joint position $\theta_{\text {ref1 } 1}$ and $\theta_{\text {ref } 2}$ are shown in Fig. 3. These desired joint positions and their derivatives will be fed to the control algorithms implemented by a PC. The initial position of the robot arm is set at $\theta_{\text {ref1 }}=60 \mathrm{deg}$. and $\theta_{\text {ref2 }}=100 \mathrm{deg}$. (angular joint coordinates), $\mathrm{x}_{0}$, $\mathrm{y}_{0}=[-0.074,0.205]$ (task space coordinate) shown in Fig. 4. Angular joint velocity $=$ $0.6 \mathrm{~m} / \mathrm{sec}$. Angular joint acceleration $=0.2 \mathrm{~m} / \mathrm{sec}^{2}$, Radius of the circle is $0.12 \mathrm{~m}$, with 1253 samples and $5 \mathrm{sec}$. running time.

\section{EXPERIMENTAL PHASE:}

The experimental phase is assumed that experimental input output data, $Z^{N}$, describing the underlying system in its entire operating region has been obtained.

$$
Z^{N}=\{[u(t), y(t)] \mid t=1, \ldots, N\}
$$


$u(t)$ is a set of inputs, driving signal to the drive motor that represent "u", Likewise $y(t)$ now represents the measured output signal $\theta_{m}$. ' $t$ ' specifies sampling instant number. Actual task space is shown in Fig. 5.

\section{MODEL STRUCTURE PHASE.}

Multilayer perceptrons neural networks are known to be an universal function approximators. The Universal Function Approximation theorem states that a single hidden layer with sigmoidal (or hyperbolic tangent) activation functions plus one layer of linear output neurons is sufficient for a multilayer perceptron to compute an approximation with a bounded error to an arbitrary continuous function [6].

Assuming that a data set has been acquired, the next step is to select a model structure. Unfortunately, this issue is much more difficult in the nonlinear case than in the linear case. Not only is it necessary to choose a set of regressors but also a network architecture is required, as we are going to use the neural network as a learning tool. The approach used here is described in [7]. The idea is to select the regressors based on inspiration from linear system identification and then determine the best possible network architecture with the given regressors as inputs. $\varphi(t)$ is a vector containing the regressors, $\theta$ is a vector containing the weights and $g$ is the function realized by the neural network.

$$
\hat{y}(t \mid \theta)=\hat{y}(t \mid t-1, \theta)=g(\varphi(t), \theta)
$$

A function, which uses a Levenberg-Marquardt algorithm is implemented to generate the model. A Levenberg-Marquardt method is the standard method for minimization of mean-square error criteria, due to its rapid convergence properties and robustness. [8].

\section{IDENTIFICATION RESULT}

A number of different model structures, before picking the best one, have been investigated. As regressors we will use two past inputs and two past outputs. Furthermore we will choose network architecture with 5 hidden tanh units and one linear output unit. Now that a model structure has been selected, we are ready to begin training. Let's run the NNARX function, which uses a Levenberg Marquardt algorithm for generating a NNARX-model.[9]. The following are W1, W2, Weight matrices 1000 samples of data are used for training the network Fig. 6, and the rest (253 samples) are used for validation.

- W1: Input-to-hidden layer weights. The matrix dimension is [(\# of hidden units)-by-(inputs + 1)] (the 1 is due to the bias).

- W2: hidden-to-output layer weights. Dimension is [(outputs) * (\# of hidden units + 1)]

The following identification is for the first arm and next, by the same way, we will identify the second arm dynamic model. 


\section{Fit a Linear Model}

The golden rule in identification (and in most other matters) is to try simple things first. If a linear model does a decent job, one should not bother wasting time on fancy neural network based model structures. To identify a linear OE model, the System Identification Toolbox from The MathWorks, Inc. is employed [10]. Fig. $7 \& 8$ show validation of linear model. From the comparison plot, as well as from the correlation functions, it, clearly, appears that the linear model has severe problems. It is thus concluded that this is due to the underlying system being nonlinear.

\section{Fit a Non Linear Model (Neural Network)}

For comparison a NNARX model structure is attempted. A fully connected 2 layers network architecture with 5 hidden hyperbolic tangent units and 1 linear output is selected. The network now has to be trained to obtain the weights w1\&w2.

\section{Validation of the trained network}

This looks quite satisfactory, and it is definitely better than the linear model. However, it is never a problem to fit the training data accurately. Redoing the validation on the test set clearly gives a less flattering result. Fig. 9 \& 10 depict validation charts for the chosen structure. Fig. 11 \& 12 depict validation results, for the optimal network architecture, after pruning neural networks model structure by applying regularization. Experience has shown that regularization is helpful when. pruning neural networks. A remarkable change in performance when using both formats, short and long. Long format has been appreciated. The correlation coefficients almost stay within their standard deviations now and thus look far better than those shown previously:

\section{CONCLUSION}

A Neural Network model structure and an OE linear model are used to identify non linear, flexible, hand made robot manipulator dynamical system.

The case has been treated as there is no particular physical knowledge about the system properties, black box case. Validation of linear model has found severe problems. It is thus concluded that this is due to the underlying system being nonlinear. Different neural network model structures ,before picking the best one, should be investigated. The model structure selection consists of two subproblems: Choosing a regressor structure and choosing a network architecture. Validation of the trained NNARX network, looks quite satisfactory, and it is definitely better than the linear model. Experience has shown that regularization is helpful when pruning neural networks. A remarkable improvement in performance, when using long instead of short format for choosing neural network weights, is appreciated. 


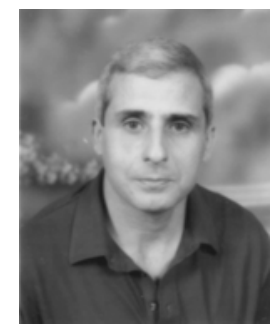

\section{REFERENCES}

[1] Spong, M. W., Lewis, F., and Abdallah, C., "Robot Control: Dynamics, Motion Planning, and Analysis", IEEE Press, 1992.

[2] Ostring, M., S. Gunnarsson and M. Norrl", "Closed loop identification of the physical parameters of an industrial robot." In: Proc. 2001, of the 32nd ISR(International Symposium on Robotics). Seoul, Korea.

[3] Lennart Ljung, "Black-box models from input-output measurements" Report no.: LiTH-ISY-R-2362, For the 18th IEEE Instrumentation and Measurement Technology Conference, Budapest, 2001]

[4] Ljung, L.. "System Identification Toolbox - For Use with Matlab". The MathWorks Inc. 2000.

[5] Mohamed M. Negm, Ashraf S. Awad, and Aziz I. Said "Digital Control of Direct Drive Robot Manipulators" in Proc. International Conference on Power System Technology -POWERCON 2004 Singapore , 21 -24 November 2004.

[6] Aziz I. Said, Mohamed M. Negm , \& Ashraf S. Awad "Performance Control Of Direct Drive Robot Manipulators For Efficient Energy Applications" in proc. Third International Conference On Energy Research And Development, November 2123, 2005, State of Kuwait.

[7] S.A. Billings, H.B., Jamaluddin, S. Chen "Properties of Neural Networks With Applications to Modelling non-linear Dynamical Systems", Int. J. Control, Vol. 55, No 1, pp. 193-224, 1992

[8] M. Nørgaard, O. Ravn, N. K. Poulsen, L. K. Hansen (2000): "Neural networks for Modelling and Control of Dynamic Systems," Springer-Verlag, London, UK, 2000.

[9] R. Fletcher (1987):"Practical Methods of Optimization", Wiley, 1987

[10]Magnus Nørgaard, "Neural Network Based System Identification TOOLBOX", Version 2, Technical Report 00-E-891, Department of Automation Technical University of Denmark, January 23, 2000

[11]L. Ljung, "System Identification Toolbox User's Guide", The MathWorks Inc., 1995.

\section{BIOGRAPHIES}

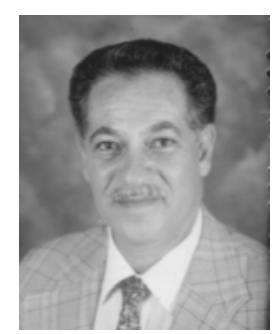

Aziz I. Said has obtained the B. Sc., M. Sc. in electrical engineering from Ain Shams University, Cairo-Egypt June 1965 and December 1969. His Ph. D. is in the field of Power Electronics and its application in May 1975 from University of Toulecese, France. His fields of interest are power electronics and its industrial applications, control in electromechanics, environmental protection, quality assurance and development of engineering education. He has worked as an expert for the Arabic and Egyptian fund of technical cooperation in Kinshasa, Congo Democratic and in Liberia for the periods 1982-1984 and 1987-1990 respectively. He is a member in many periodical journals and in many engineering associations.

Ashraf S. Awad he received the B. Sc., High Diploma, and M. Sc. in electrical power and machine, Cairo, Egypt, In 1978, 1990, and 1995, respectively. He is currently 
with Egyptian tank plant military factory 200 , machining quality production. His fields of interests include switchgear \& protection, Optimal, adaptive \& preview control, Robotics, and Laser Tracker for machining inspection and control.

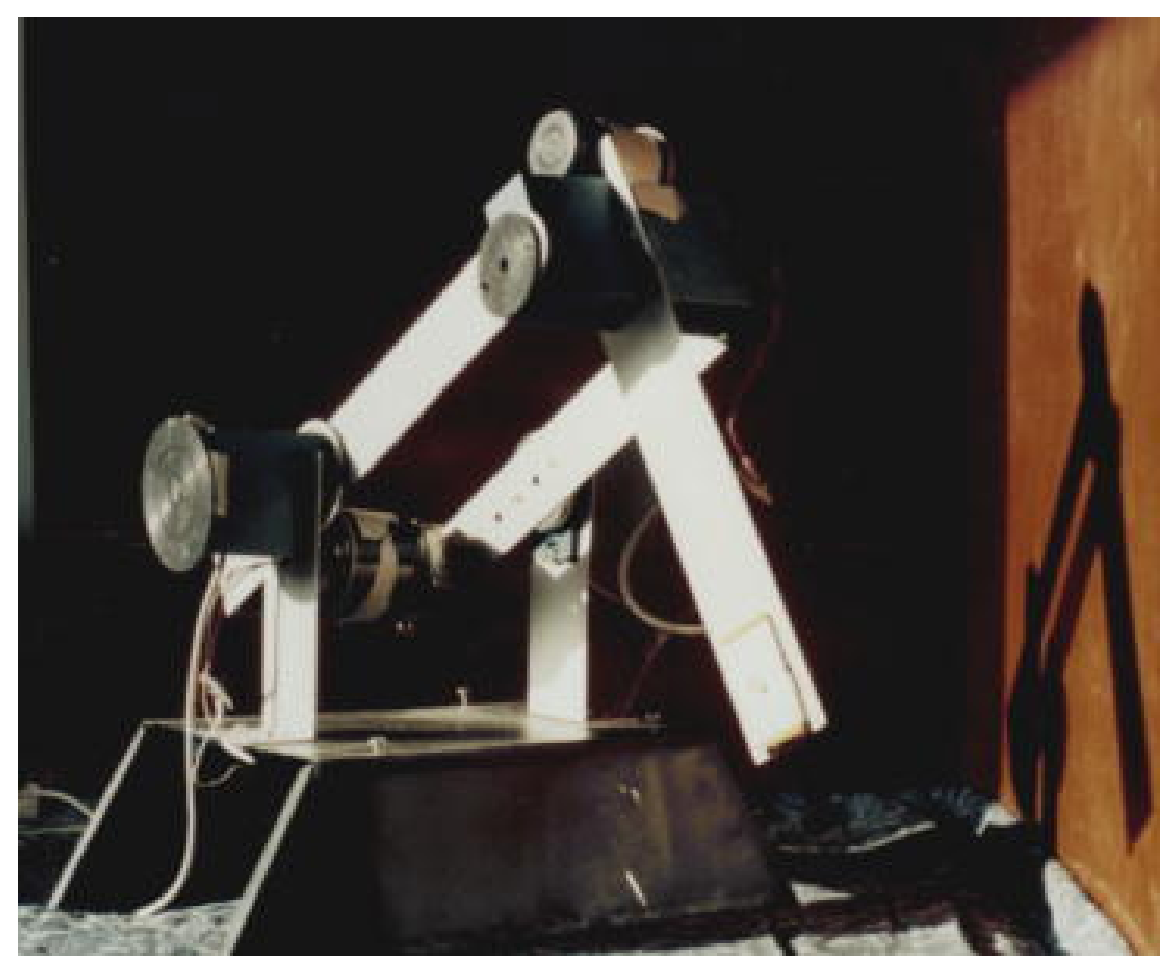

Fig. 1, Experimental robot used in the identification

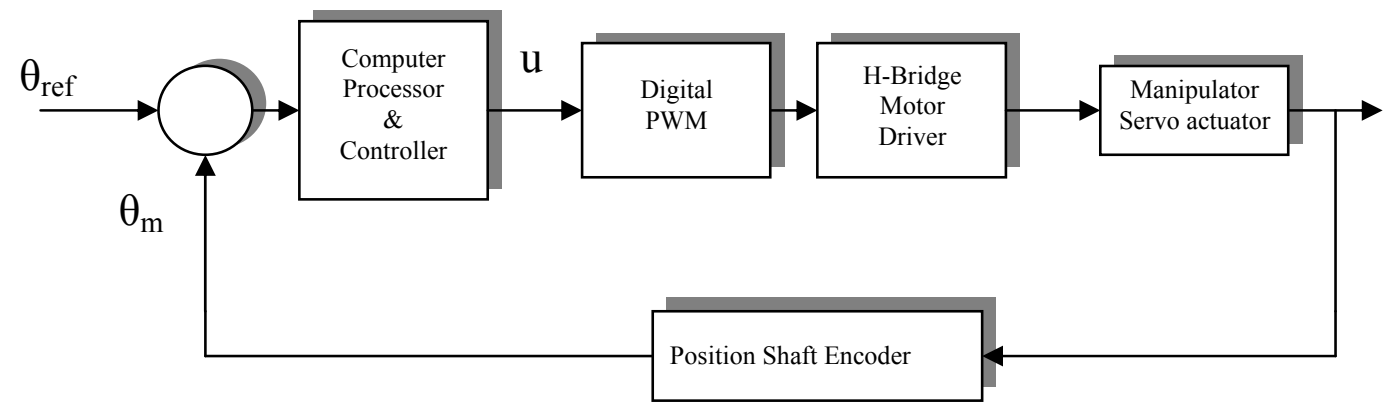

Fig. 2. Block diagram of the robot system 

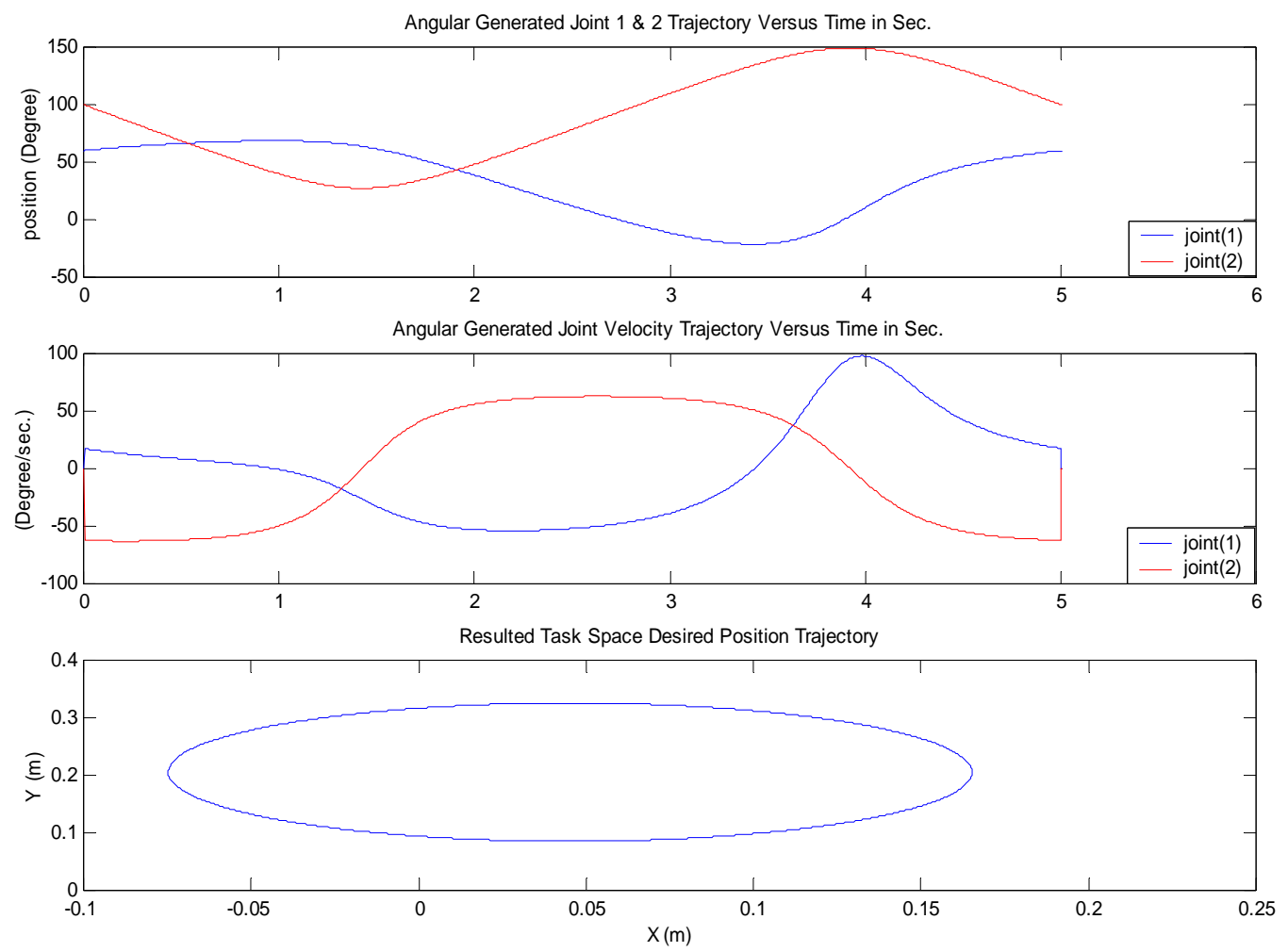

Fig. 3. Circular shape angular joint position, velocity, and $x-y$ task space trajectory
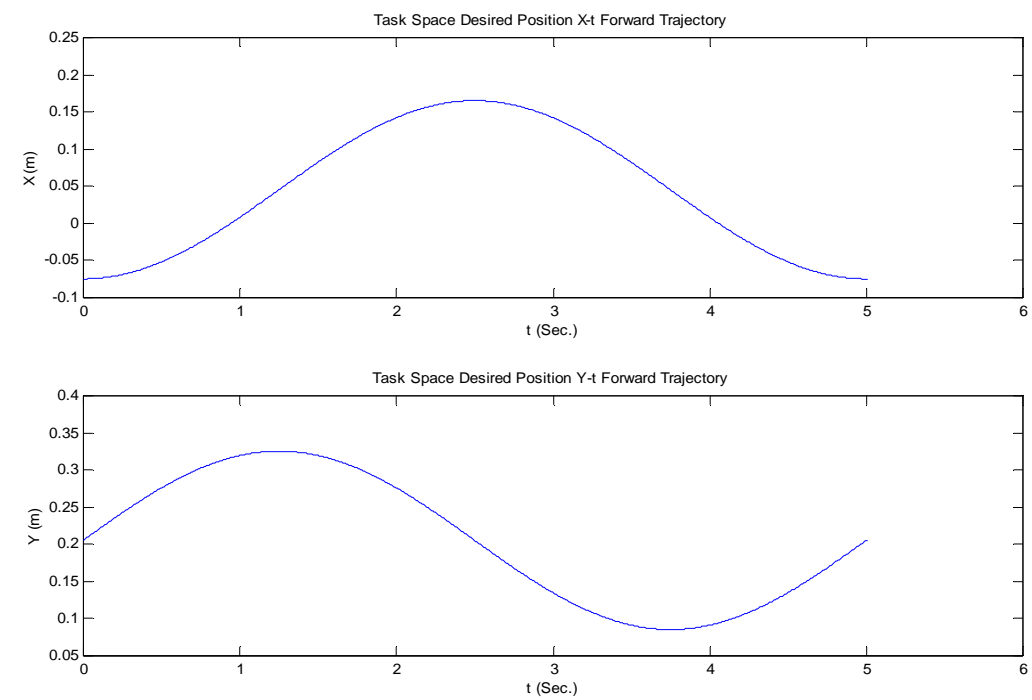

Fig.. 4. Circular shape $x-y$ versus time trajectory 


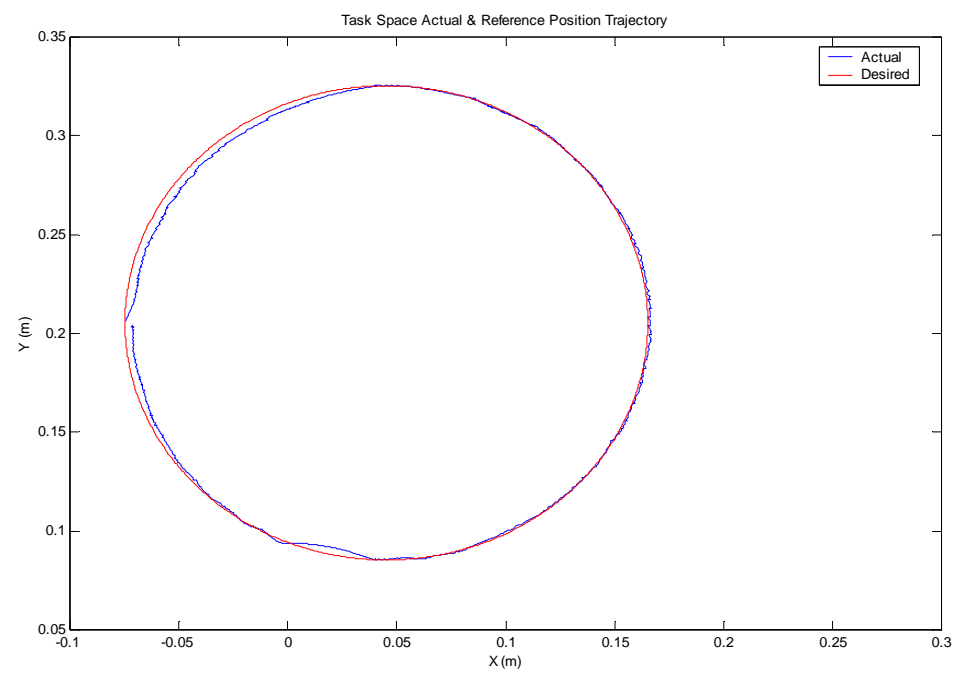

Fig. 5. Actual circular task space versus reference trajectory
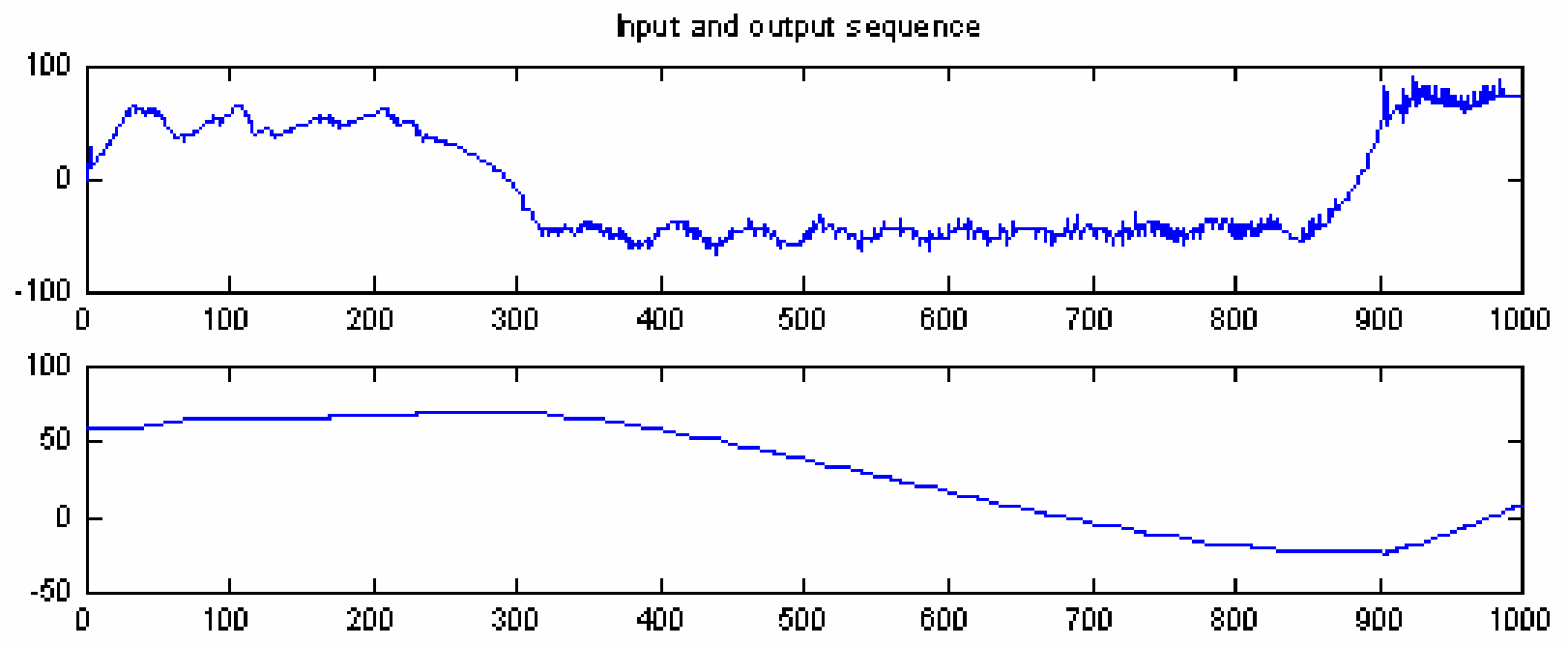

Fig. 6, Input and output data (first 1000 samples.

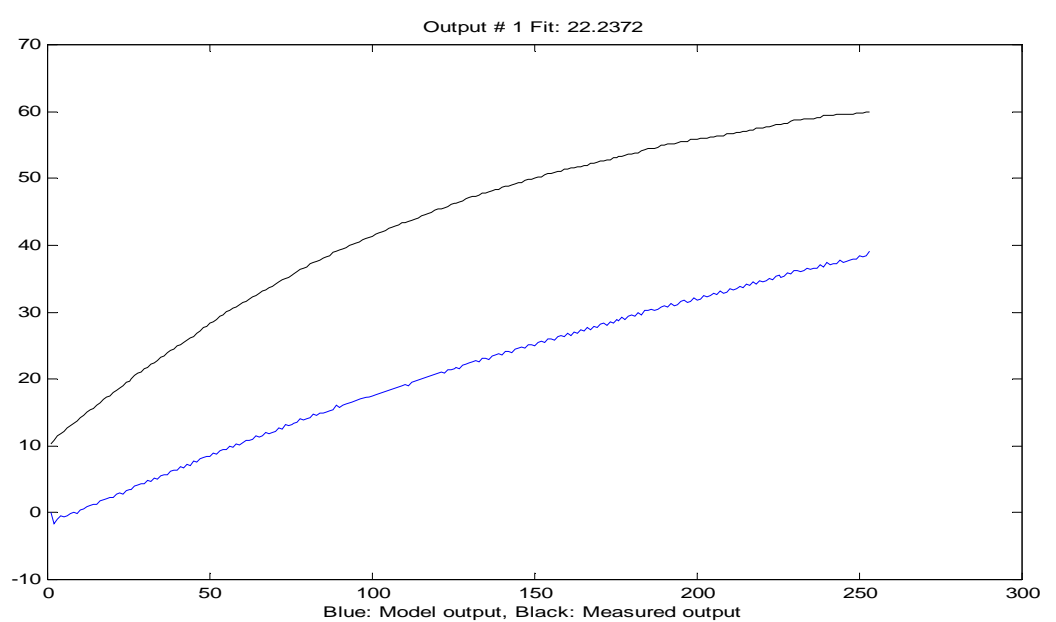

Fig. 7, Actual model measured versus identification model output 

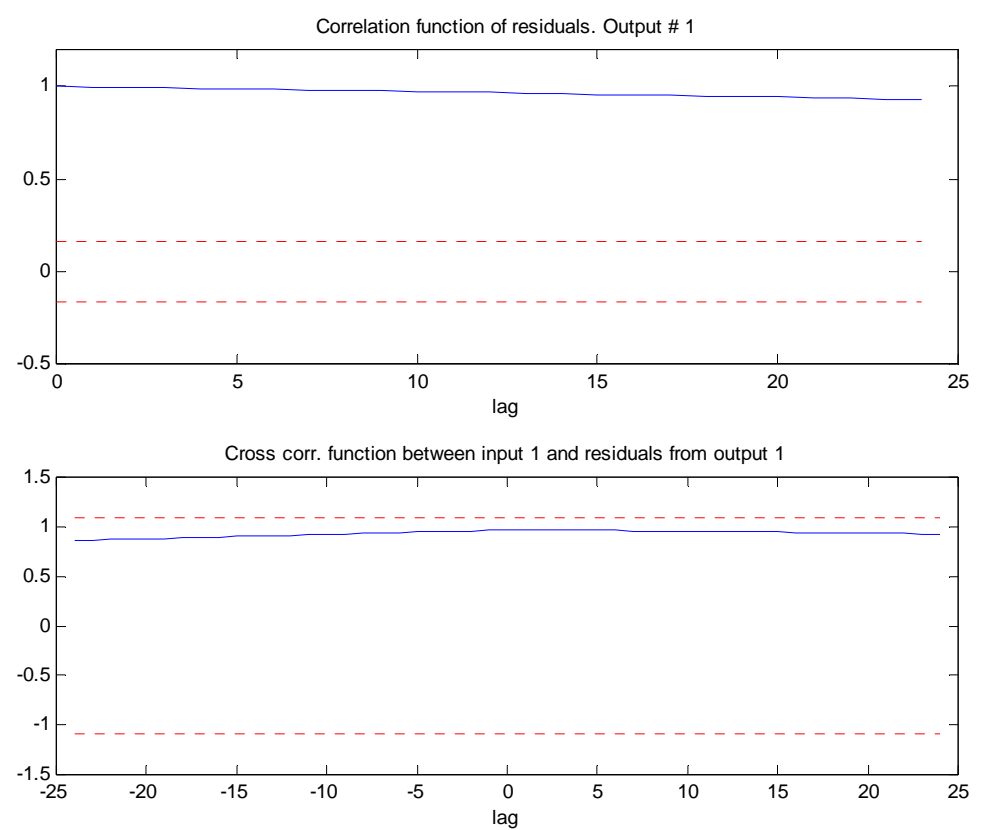

Fig. 8, Correlation and cross correlation of residuals.
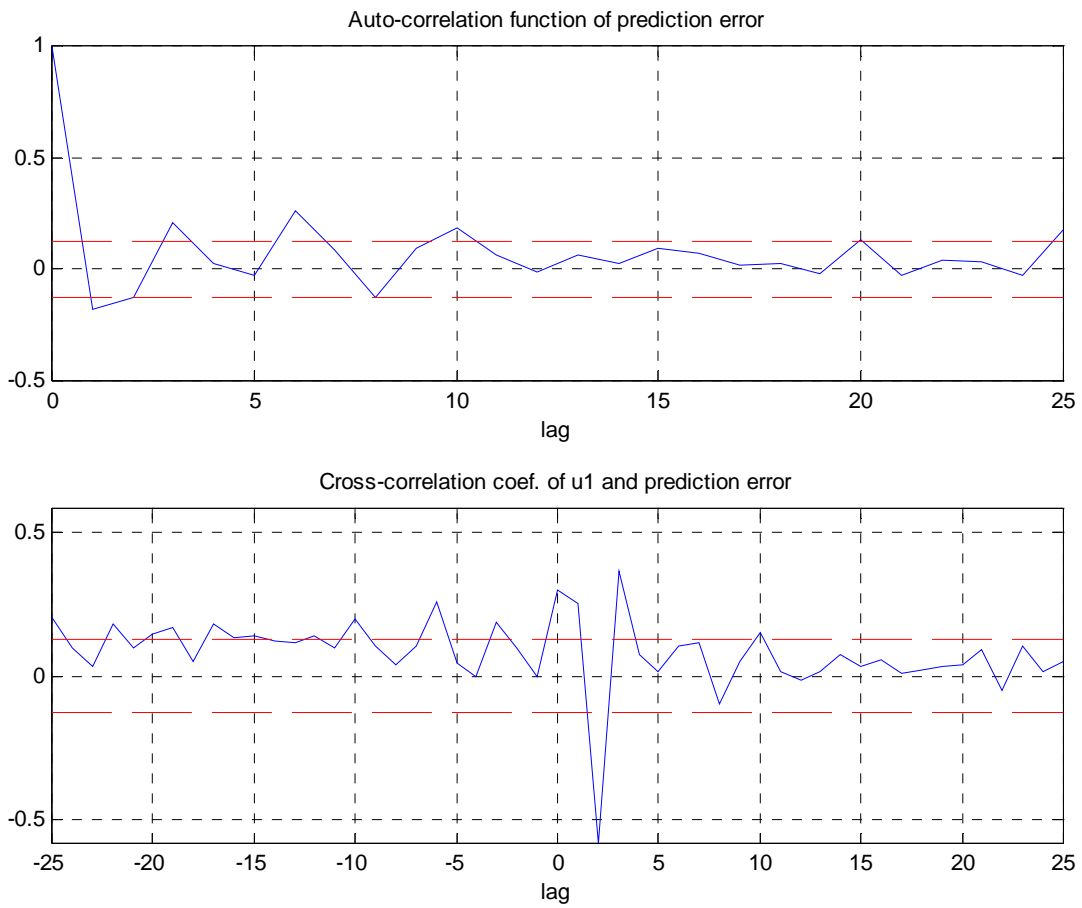

Fig, 9, NNARX model, correlation of prediction error (up chart) and cross-correlation of $\mathrm{u}_{1}$ and prediction error (down chart). 

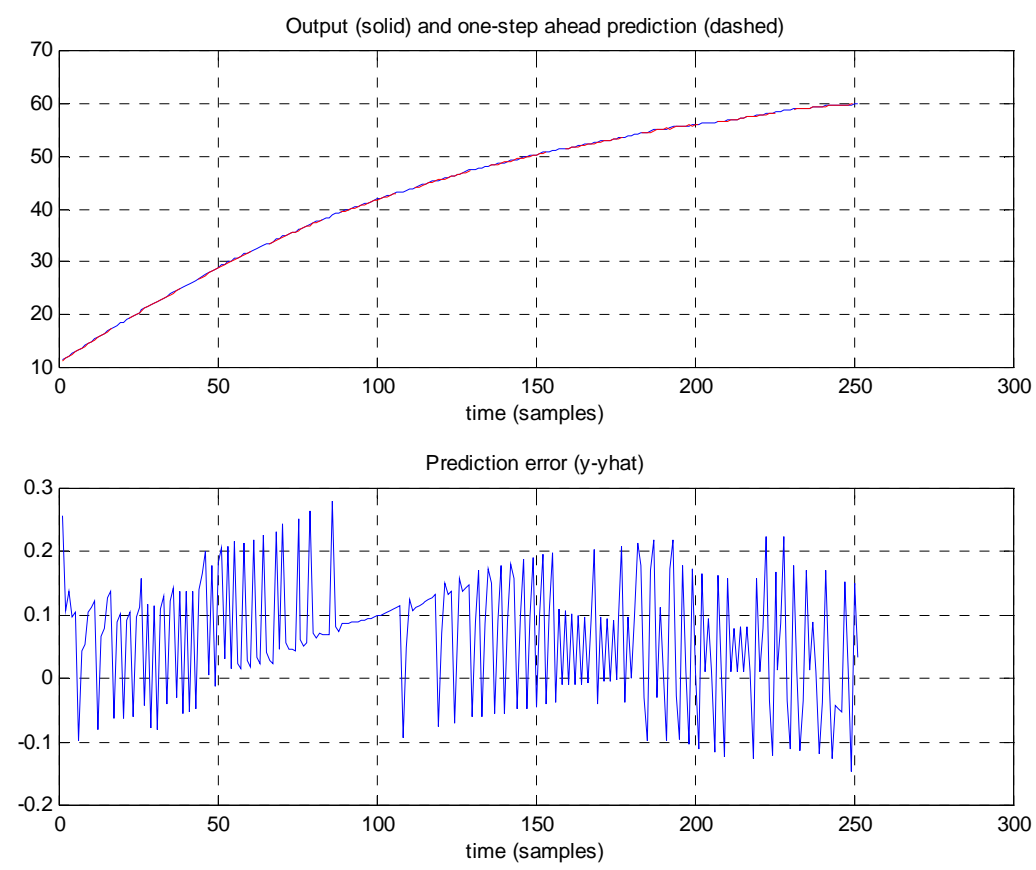

Fig. 10, Actual and NNARX prediction output (up chart), and residuals (down chart).
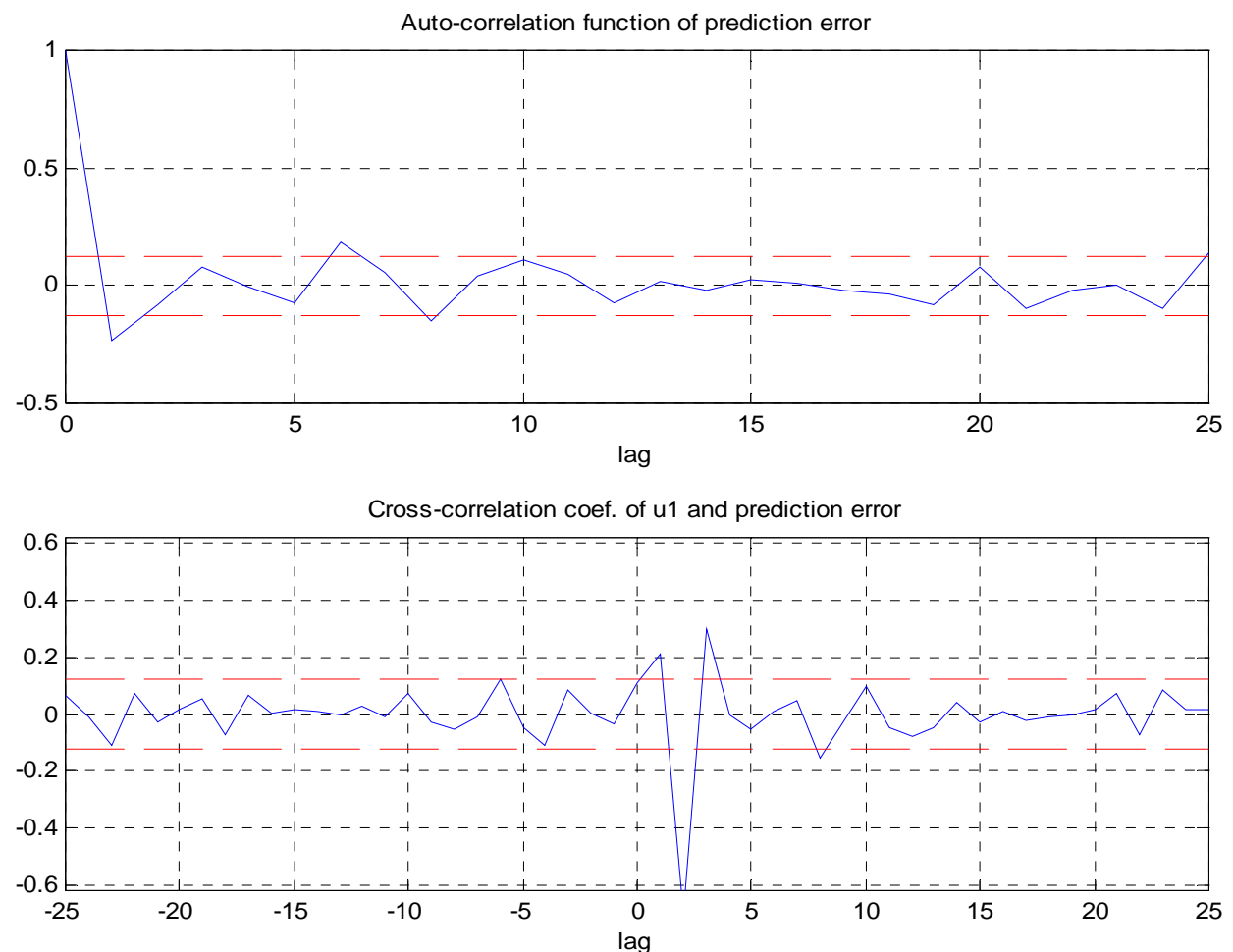

Fig. 11, Regularization model, correlation of error (up) and cross-correlation of $\mathrm{u}_{1}$ and prediction error (down chart). 

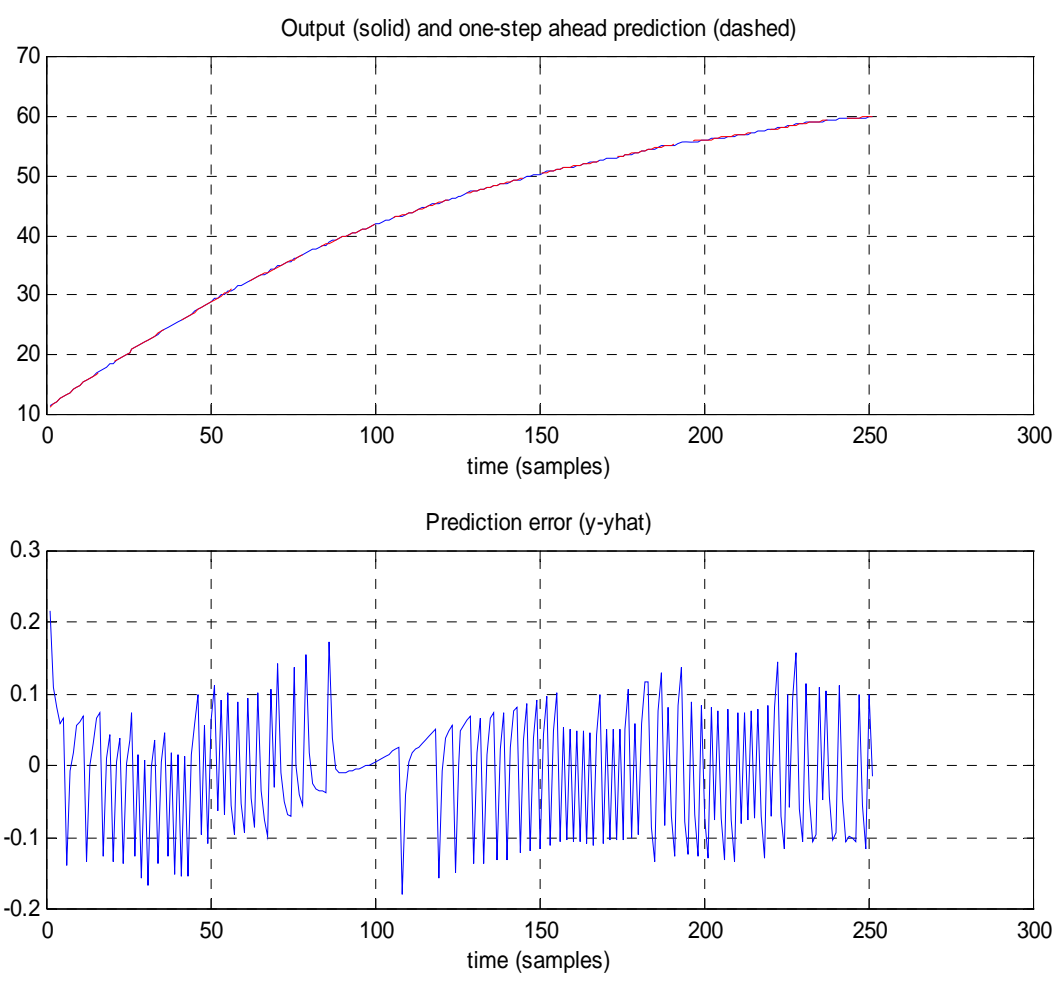

Fig. 12, Actual and NNARX, regularization model, prediction output (up chart), and residuals (down chart). 